\title{
Controlled Propagation of Spiking Dynamics in Vertical-Cavity Surface-Emitting Lasers: Towards Neuromorphic Photonic Networks
}

\author{
Tao Deng, Joshua Robertson and Antonio Hurtado
}

\begin{abstract}
We report experimentally and in theory on the controllable propagation of spiking regimes between two interlinked Vertical-Cavity Surface-Emitting Lasers (VCSELs). We show that spiking patterns generated in a first transmitter VCSEL (T-VCSEL) are communicated to a second receiver VCSEL (R-VCSEL) which responds by firing the same spiking response. Importantly, the spiking regimes from both devices had analogous temporal and amplitude characteristics, including equal number of spikes fired, same spike and inter-spike temporal durations and similar spike intensity properties. These responses are analogous to the spiking communication patterns of biological neurons yet at sub-nanosecond speeds, this is several (up to 8) orders of magnitude faster than the timescales of biological neurons. We have also carried out numerical simulations reproducing with high degree of agreement the experimental findings. These results obtained with inexpensive, commercially available VCSELs operating at important telecom wavelengths $(1300 \mathrm{~nm})$ offer great prospects for the scaling of emerging VCSEL-based photonic neuronal models into network configurations for use in novel neuromorphic photonic systems. This offers high potentials for non-traditional computing paradigms beyond digital systems and able to operate at ultrafast speeds.
\end{abstract}

Index Terms - vertical-cavity surface-emitting lasers (VCSELs), neuromorphic photonics, photonic neurons, spiking, photonic spiking processing.

\section{INTRODUCTION}

$\mathrm{N}$ EUROMORPHIC computing aims at emulating the powerful computational features of the brain to develop novel information processing systems beyond traditional digital models [1]. Exploring the activity mechanism of biological neurons and emulating their complex computational capabilities becomes therefore an area of great interest. Traditionally, electronic implementation of neuronal models were studied [1-6] yielding nowadays realization of computing architectures such as the Neurogrid at Stanford University [7], Truenorth at IBM [8] and the Neuromorphic Chip at the

Manuscript received on January 20th, 2017. This work was supported by the National Natural Science Foundation of China under Grant 61674123, the Natural Science Foundation of Chongqing City under Grant 2016jcyjA0082 and the University of Strathclyde under the Strathclyde's Chancellor Fellowships and RI@S programmes.

T. Deng is with the School of Physical Science and Technology, Southwest University, Chongqing 400715, China, and also with the Institute of Photonics,
University of Manchester [9], among others. These works have revealed the huge potential of neuromorphic technology and the revolutionary progress that can trigger in future information processing modules. Yet, although these aforementioned approaches are effective to target the millisecond time scales of biological neurons they have still important limitation to yield higher frequency operation [10].

Neuromorphic photonics has recently emerged offering great prospects for novel neuronal models operating at ultrafast speeds, up to 9 orders of magnitude faster than biological neurons. This paves radically new paths to break out the bandwidth limitation of actual neuromorphic processors [1024]. In recent years, the number of works exploring different neuro-inspired photonic approaches have multiplied (for a review see [12] and references therein). Among these, approaches based on different semiconductor lasers (SLs) have clearly dominated $[12,16]$ given the potentials of these devices for application in information processing [15-22], optical interconnects [23] and communication networks [24]. Different techniques considering micro-ring [13] and Quantum Dot SLs [17,24], devices with saturable absorbing sections [22], and coupled to photodiodes [10], etc. have been used to produce neuron-inspired spiking patterns using either optical feedback and optical injection techniques (see [12]). Moreover, works investigating interconnected systems based on quantum-well lasers [25], quantum-dot lasers [26], microdisk lasers [27], microrings [28] and modulators and nonlinear loop mirrors [11] for neuromorphic applications have also been recently reported. As a result, combining neuronal concepts with SLs opens new routes for neuro-inspired photonic modules in information processing tasks such as clock recovery, pulse reshaping, and ultrafast neuromorphic photonic computation.

Amongst SL, Vertical-Cavity Surface-Emitting Lasers (VCSELs) have some unique advantages, e.g. low costs, easyintegration into 2D and 3D arrays, and high coupling efficiency to optical fibers [29-31], etc. Correspondingly, the investigation of VCSEL-based photonic neuronal models is of particular interest given their special attributes and high prospects for scaling into network configurations. Recently, high-amplitude

SUPA Dept. of Physics, University of Strathclyde, TIC Centre, 99 George Street, Glasgow G1 1RD, United Kingdom (e-mail: dengt@swu.edu.cn).

J. Robertson and A. Hurtado are with the Institute of Photonics, SUPA Dept. of Physics, University of Strathclyde, TIC Centre, 99 George Street, Glasgow G1 1RD, United Kingdom (e-mail: antonio.hurtado@strath.ac.uk). 
self-generated [32] and controllable produced excitable spikes [33-35] have been reported in optically injected VCSELs. Furthermore, applications of these spiking dynamics for information processing tasks has been proposed [36-43]. Also, in a recent work we have demonstrated the controllable and reproducible achievement of diverse spiking patterns in longwavelength VCSELs $[35,42,43]$ outlining their potentials for all-optical binary-to-spiking format conversion applications at telecom wavelengths. Whilst these early reports have shown the possibilities of VCSELs (and SLs in general) for novel photonic neuronal models, the majority only discussed the spiking characteristics of single elements. Yet, the experimental demonstration of network characteristics with laser-based photonic neurons is strictly necessary if neuromorphic photonic systems are to be used in novel functional information processing architectures.

This work focuses on this key challenge investigating the propagation of spiking information between two interconnected VCSEL photonic neurons. Here, we experimentally demonstrate a spiking propagation system based on two unidirectionally coupled $1300 \mathrm{~nm}$ VCSELs. A numerical model is also developed to analyze in theory the spiking propagation characteristics of such system showing very good agreement with the experimental findings. We show that controllable and reproducible spiking patterns induced in the first transmitter VCSEL (T-VCSEL) in response to incoming perturbations are successfully propagated to the second receiver VCSEL (RVCSEL). Moreover, the transmitted and received spiking patterns in the interconnected system can be simply controlled by adjusting the intensity and duration of short-temporal perturbations encoded in the external injected signals.

\section{EXPERIMENTAL SETUP}

Fig.1 (a) shows the schematic diagram of our experimental setup. Two commercially-available fibre-coupled $1300 \mathrm{~nm}$ VCSELs were used (T- \& R-VCSELs) in this work. The optical spectra of the two devices are respectively plotted in figs. 2(a) and 2(b) showing in both cases emission into two modes. These corresponded to the two orthogonal polarizations of the fundamental transverse mode of the devices. Throughout this work we refer to the polarization of the main lasing mode of the devices as parallel polarization, whereas the polarization of attenuated longer wavelength mode is referred as orthogonal polarization. During the experiments, the temperatures of both VCSELs were kept constant at all moments at $300 \mathrm{~K}$ (TVCSEL) and 292K (R-VCSEL) respectively. This slight difference in operating temperatures was set in order to match the emission wavelengths from both VCSELs. At the temperatures configured the threshold currents for both VCSELs were measured equal to $I_{t h, T}=0.60 \mathrm{~mA}$ and $I_{t h, R}=0.61$ $\mathrm{mA}$. Additionally, the applied bias currents were set equal to $1.5 \mathrm{~mA}$ (about $2.5 I_{t h, T}$ ) for T-VCSEL and $1.5 \mathrm{~mA}$ (about 2.5 $\left.I_{t h, R}\right)$ for R-VCSEL, respectively.

Light from a Tunable Laser (TL) source was injected into TVCSEL. The output of the TL was externally modulated using a Mach-Zehnder (MZ) modulator and a signal generator (SG) to add temporal perturbations in the form of short power drops and with durations ranging from $0.8 \mathrm{~ns}$ to $6.8 \mathrm{~ns}$. Fig. 2(c) shows a typically injected signal. This has a constant injection level $K_{i n j}$, and added perturbations with controlled strength $K_{p}$ (defined as the ratio between $K$ and $K_{i n j}$, namely, $K_{p}=K / K_{i n j}$ ) and temporal duration $t_{d}$. The repetition rate between encoded perturbations was $f_{\text {rep }}=15 \mathrm{MHz}$ and the time delay between $\mathrm{T}$ VCSEL and R-VCSEL was approximately equal to $62 \mathrm{~ns}$. The external signal was set with linear orthogonal polarization and injected into the subsidiary mode of T-VCSEL via a first optical circulator (OC1). The reflective output from T-VCSEL was collected from $\mathrm{OC} 1$ and divided into two parts by means of a fiber directional coupler (FC1). The $90 \%$ port from $\mathrm{FC} 1$ is injected into second VCSEL (R-VCSEL) after going through an optical isolator (ISO), a variable attenuator (VA), another polarization controller (PC3) and a second optical circulator (OC2). PC3 was used to set the light from T-VCSEL with linear orthogonal polarization prior to its injection into the subsidiary mode of R-VCSEL. The $10 \%$ port from FC1 is sent to the detection system to analyze the dynamics of the signal coming from T-VCSEL. Similarly, the reflective output from RVCSEL was collected from OC2 and sent for analysis. The detection system was formed by an optical spectrum analyzer (OSA) and two $12 \mathrm{GHz}$ amplified photodetectors coupled to a $13 \mathrm{GHz}$ real time oscilloscope to capture real-time traces from T- and R-VCSELs. A power meter (PM) was also used to monitor the injection power into the two VCSELs of this work.

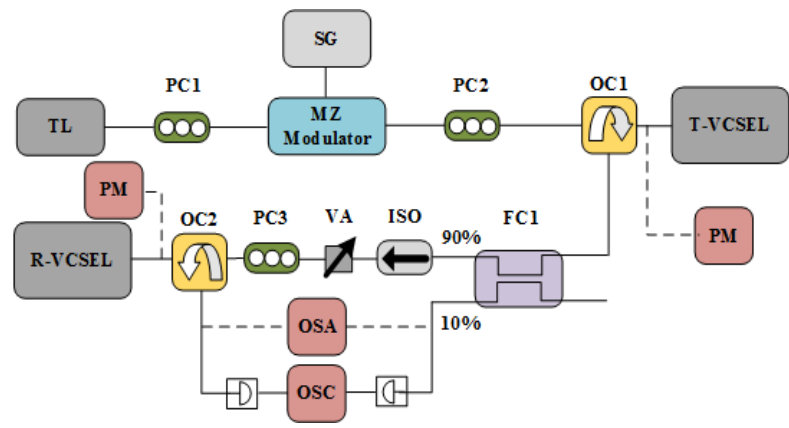

Fig. 1. Experimental setup. TL: tunable laser; T-VCSEL: transmitter-VCSEL; R-VCSEL: receiver-VCSEL; PC: polarization controller; MZ: Mach-Zehnder Modulator; SG: signal generator; FC: fiber coupler; OC: optical circulator; ISO: isolator; VA: variable attenuator; PM: power meter; OSA: optical spectrum analyzer; OSC: oscilloscope.
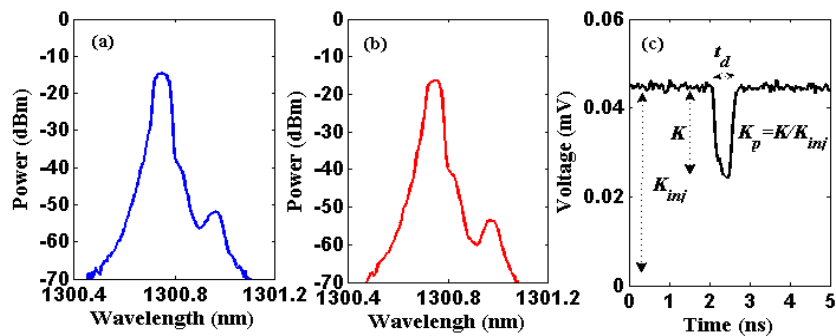

Fig. 2. Optical spectra of (a) T-VCSEL and (b) R-VCSEL biased at 1.5mA. (c) Injected signal with a constant input level $K_{i n j}$ and added perturbations (power drops) with relative intensity $K_{p}$ and temporal duration $t_{d}$.

\section{THEORY}

In this work we have used an extension of the wellestablished spin-flip model (SFM) to numerically simulate the 
propagation characteristics of spiking dynamics in two interconnected VCSELs. Using this model the rate equations for T- and R-VCSEL can be described by [40,41]:

$$
\begin{aligned}
\frac{d E_{x, y}^{T}}{d t}= & -\left(k^{T} \pm \gamma_{a}^{T}\right) E_{x, y}^{T}-i\left(k^{T} \alpha^{T} \pm \gamma_{p}^{T}\right) E_{x, y}^{T} \\
& +k^{T}\left(1+i \alpha^{T}\right)\left(N^{T} E_{x, y}^{T} \pm i n^{T} E_{y, x}^{T}\right) \\
& +\eta_{1} E_{\text {injx,injy }}(t) e^{i \Delta \omega_{x, y} t}+F_{x, y}^{T} \\
\frac{d E_{x, y}^{R}}{d t}= & -\left(k^{R} \pm \gamma_{a}^{R}\right) E_{x, y}^{R}-i\left(k^{R} \alpha^{R} \pm \gamma_{p}^{R}\right) E_{x, y}^{R} \\
& +k^{R}\left(1+i \alpha^{R}\right)\left(N^{R} E_{x, y}^{R} \pm i n^{R} E_{y, x}^{R}\right) \\
& +\eta_{2} E_{x, y}^{T}(t) e^{-i \omega_{x, y}^{T} \tau+i \Delta \omega_{T R} t}+F_{x, y}^{R} \\
\frac{d N^{T, R}}{d t}= & -\gamma_{e}^{T, R}\left[N^{T, R}\left(1+\left|E_{x}^{T, R}\right|^{2}+\left|E_{y}^{T, R}\right|^{2}\right)-\mu^{T, R}\right. \\
& \left.+i n^{T, R}\left(E_{y}^{T, R} E_{x}^{T, R^{*}}-E_{x}^{T, R} E_{y}^{T, R^{*}}\right)\right] \\
\frac{d n^{T, R}}{d t}= & -\gamma_{s}^{T, R} n^{T, R}-\gamma_{e}^{T, R}\left[n^{T, R}\left(\left|E_{x}^{T, R}\right|^{2}+\left|E_{y}^{T, R}\right|^{2}\right)\right. \\
& \left.+i N^{T, R}\left(E_{y}^{T, R} E_{x}^{T, R^{*}}-E_{x}^{T, R} E_{y}^{T, R^{*}}\right)\right]
\end{aligned}
$$

where superscripts $T$ and $R$ stand for T-VCSEL and R-VCSEL, respectively, and subscripts $x$ and $y$ stand for $\mathrm{X}$ - (or orthogonal) and $\mathrm{Y}$ - (or parallel) polarization outputs. $E$ is the slowly varying complex amplitude of the VCSEL's output field. $E_{\text {injx }}\left(E_{\text {injy }}\right)$ is the slowly varying complex amplitude of the injected light field into the $\mathrm{X}-(\mathrm{Y}-)$ polarization components. $N$ is the total population inversion between conduction and valence bands, $n$ is the difference between the population inversions for spin-up and spin-down radiation channels, respectively. $k$ is the decay rate of the electric field in the VCSEL cavity. $\alpha$ is the linewidth enhancement factor, $\gamma_{e}$ is the decay rate of the total population inversion $N$ and $\gamma_{s}$ is the spin-flip relaxation rate. $\gamma_{a}$ and $\gamma_{p}$ are respectively the linear dichroism and the cavity birefringence rate. $\tau$ is the optical flight time from T-VCSEL to R-VCSEL and $\mu$ is the normalized injection current. $\Delta \omega_{x}\left(\Delta \omega_{y}\right)$ indicates the detuning between the angular frequency of the x-polarized (or y-polarized) externally injected light $\omega_{\text {injx }}\left(\omega_{\text {injy }}\right)$ and a reference angular frequency situated at the middle point between those of the VCSEL's X- and Y-polarization outputs, i. e. $\Delta \omega_{x}=\omega_{i n j x}-\left(\omega_{x}{ }^{T}+\omega_{y}{ }^{T}\right) / 2\left(\Delta \omega_{y}=\omega_{i n j y}-\left(\omega_{x}{ }^{T}+\omega_{y}{ }^{T}\right) / 2\right) . \Delta \omega_{T R}$ refers to the frequency detuning between the frequencies of the two VCSELs: T-VCSEL $\left(\omega^{T}\right)$ and R-VCSEL $\left(\omega^{R}\right)$, i. e. $\Delta \omega_{T R}=$ $\omega^{T}-\omega^{R} . \eta_{1}$ and $\eta_{2}$ are the injected coupling coefficient for TVCSEL and R-VCSEL, respectively. $F$ corresponds to the spontaneous emission noise and the expressions is given by:

$$
\begin{aligned}
F_{x}^{T, R}= & \sqrt{\frac{\beta_{s p}^{T, R} \gamma_{e}^{T, R}}{2}}\left(\sqrt{N^{T, R}+n^{T, R}} \xi_{1}^{T, R}+\sqrt{N^{T, R}-n^{T, R}} \xi_{2}^{T, R}\right) \\
F_{y}^{T, R}= & -i \sqrt{\frac{\beta_{s p}^{T, R} \gamma_{e}^{T, R}}{2}}\left(\sqrt{N^{T, R}+n^{T, R}} \xi_{1}^{T, R}\right. \\
& \left.-\sqrt{N^{T, R}-n^{T, R}} \xi_{2}^{T, R}\right)
\end{aligned}
$$

where $\beta_{s p}$ is the strength of the spontaneous emission, $\xi_{1}$ and $\xi_{2}$ are independent complex Gaussian white noise sources of zero mean and a unit variance. The rate equations (1)-(6) can be numerically solved using the fourth-order Runge-Kutta method. For simplicity, during calculations, the normalized injection current and all the internal parameters for T-VCSEL and R-VCSEL are assumed to be identical, given that both devices are equal in structure, are from the same manufacturer and have almost exactly equal performance and emission properties. The parameter values have been extracted from the literature [44,45] and are as follows: $\alpha=2, k=185 \mathrm{~ns}^{-1}, \gamma_{e}=0.5$ $\mathrm{ns}^{-1}, \gamma_{s}=110 \mathrm{~ns}^{-1}, \gamma_{a}=2 \mathrm{~ns}^{-1}, \beta_{s p}=10^{-6}, \gamma_{p}=128 \mathrm{~ns}^{-1}, \tau=62 \mathrm{~ns}$ and the central frequency of VCSEL is $1.45 \times 10^{15} \mathrm{rad} / \mathrm{s}$ (corresponding to the central wavelength of the VCSEL at 1300 $\mathrm{nm})$. The injection coefficient were set equal to $\eta_{1}=125 \mathrm{~ns}^{-1}$ and $\eta_{2}=100 \mathrm{~ns}^{-1}$ respectively.

\section{RESUlts AND DisCUSSION}

Fig. 3 (a) shows measured real-time series at the output of TVCSEL when subject to the injection of the signal in the inset above fig. 3(a). The latter (depicted in black) was characterized by a constant power level of $K_{i n j}=60 \mu \mathrm{W}$ with added perturbations of temporal duration $t_{d}=0.8 \mathrm{~ns}$ and strength of $K_{p}$ $=0.46$. This externally injected signal was set at a frequency detuning of $\Delta f=f_{i n j}-f_{T y}=-3.55 \mathrm{GHz}$, where $f_{i n j}$ and $f_{T y}$ correspond to the frequencies of the injection signal and that of the orthogonal polarization mode of T-VCSEL. The output signal from T-VCSEL, included in fig. 3(a) (blue plot), and which also becomes the input signal to R-VCSEL, was measured to have a constant power level of $K_{i n j}=52 \mu \mathrm{W}$. This was injected into the orthogonal polarization mode of RVCSEL at a frequency detuning equal to $\Delta f_{T R}=f_{T y}-f_{R y}=-$ $2.84 \mathrm{GHz}$, being $f_{R y}$ the frequency of free-running R-VCSEL's orthogonal polarization mode. Fig. 3(b) plots (in red) the measured time-series generated by R-VCSEL in response to the incoming signal from T-VCSEL. It should be pointed out the polarization of the signals entering both T-VCSEL and RVCSEL were set with orthogonal polarization and injected into the devices' subsidiary attenuated orthogonally-polarized modes. The externally injected signals into T-VCSEL and RVCSEL were also set with a suitable negative frequency detuning during our experiments [46,47].

Fig. 3(a) shows that when no perturbation is present the constant injected power $\left(K_{i n j}\right)$ is enough to cause the orthogonal polarization mode of T-VCSEL to injection-lock to the externally injected signal producing also polarization switching $[41,42]$ at the device's output. As a result of this process, a stable constant output is obtained from T-VCSEL. This response is kept until the arrival of the encoded perturbation, following which T-VCSEL fires a single sub-nanosecond spike. Once the perturbation is removed, T-VCSEL's output returns to its previous constant power level [35]. This behavior is caused by the perturbation briefly bringing T-VCSEL out of the injection locking state [20] and as a result of this locking/unlocking transition T-VCSEL reacts by firing an excitable spike [34]. This is indeed an analogous behavior to 

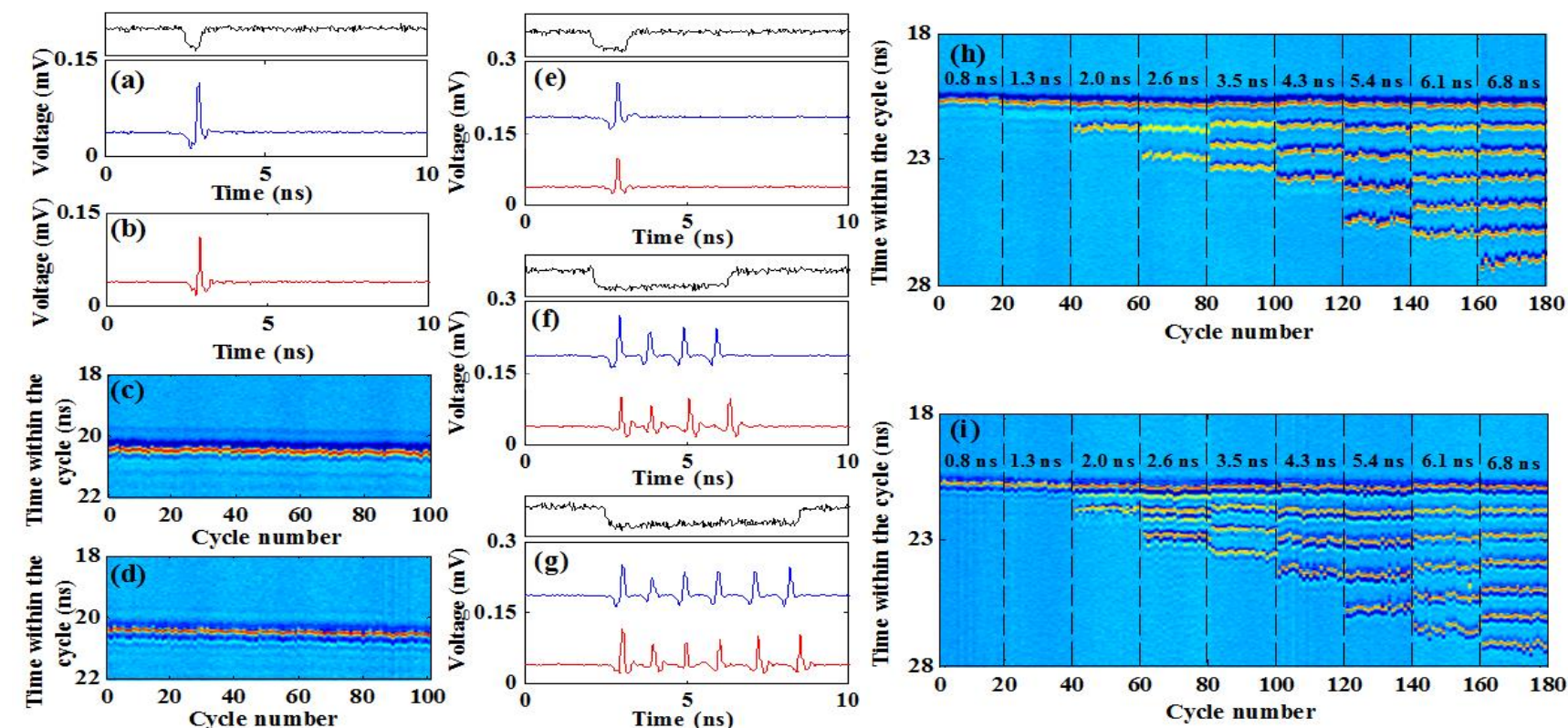

Fig.3. (a-b) Time series and (c-d) temporal maps measured at the output of T-VCSEL (a, c) and R-VCSEL (b, d).The inset at the top shows the externally injected signal into T-VCSEL characterized by a constant level of $K_{i n j}=60 \mu \mathrm{W}$ and an added perturbations with $t_{d}=0.8 \mathrm{~ns}$ and $K_{p}=0.46$. (e-g) Time series measured at the output of T-VCSEL (top) and R-VCSEL (bottom) for different values of $t_{d}$ for the perturbations arriving into T-VCSEL, namely, (e) $1.3 \mathrm{~ns}$, (f) $4.3 \mathrm{~ns}$, (g) $6.1 \mathrm{~ns}$. (hi) Temporal maps measured at the outputs of T-VCSEL (h) and R-VCSEL (i) merging results obtained for different values of $t_{d}$, from $0.8 \mathrm{~ns}$ to $6.8 \mathrm{~ns}$ as indicated and plotting the response of both VCSELs to the arrival of 20 consecutive external perturbations into T-VCSEL.

that observed in biological neurons but at much faster operation speeds [11]. Hence, as it happens in neurons in the brain our VCSEL-neuron also converts an external incoming signal into a spiking signal output, where the generated spikes precisely compute the arrival of external stimuli (perturbations).

The spiking signals generated in biological neurons are transmitted through their axons and dendrites to neighboring neurons and communicated across neuronal circuits/networks in the brain. Here, we investigate the same phenomenon by propagating the signals from T-VCSEL to a second photonic neuron in our system, R-VCSEL. Fig. 3(b) plots time series generated by R-VCSEL in response to incoming signals from T-VCSEL. As fig. 3(b) shows the output signals from RVCSEL have remarkably the same features to those from TVCSEL, thus providing a first demonstration of the successful propagation of spiking activity at sub-nanosecond speeds between two interconnected VCSEL-neurons.

The observed behavior can be explained as follows: the constant injection level coming from T-VCSEL's signal $(52 \mu \mathrm{W})$ is strong enough to cause the orthogonal polarization mode of R-VCSEL to injection lock to T-VCSEL. Hence, it reacts generating also a constant power output. The arrival of the spike generated by T-VCSEL in response to the external perturbation produces also an unlocking/locking transition in RVCSEL which makes R-VCSEL to respond firing a similar subnanosecond spike. It should be noted that for simplicity the time series of R-VCSEL have been shifted to be plotted in the same time-scales as those from T-VCSEL. However, the spike generated by R-VCSEL is obtained $\sim 62$ ns later than that from T-VCSEL, where this time corresponds to the optical flight time between the two devices. This value can be easily controlled by experimentally increasing (or reducing) the length of the optical link separating both elements. This is important as it opens new possibilities for the use of the time difference between spiking events in interconnected elements as an extra computational feature, much as biological neurons do in the brain.

Fig. 3(c) and Fig. 3(d) show respectively the corresponding experimental (colour-coded) temporal maps plotting superimposed time series captured at the outputs of T-VCSEL and R-VCSEL. In both cases, the time interval between two consecutive events $\left(T_{\text {rep }}=1 / f_{\text {rep }}\right)$ is used as the folding parameter [35]. These maps allows us to depict in a single plot the response of the system to a high number of consecutive arriving events (external perturbations) which for the case of figs. 3(c) and $3(\mathrm{~d})$ is 100 . The color coding in the maps indicate increasing intensity from blue to red. Light blue color corresponds to a constant output level whilst darker blue indicates power drops below the steady state and red/yellow colors correspond to spikes fired in the system. The map in fig. 3(c) clearly shows that the same spiking response is obtained for every single one of the 100 incoming perturbations into TVCSEL. This is graphically shown from the single straight line propagating undisturbed in the map of fig. 3(c). In turn, fig. 3(d) shows a similar behavior where the same pattern is also observed at the output of R-VCSEL in response to the 100 consecutive incoming spikes from T-VCSEL. Hence, controllable and reproducible spiking responses can be obtained first from a VCSEL-neuron upon the arrival of external stimuli (T-VCSEL) and this spiking information can be successfully propagated to a second VCSEL-neuron in a circuit (R-VCSEL). This result opens indeed exciting new routes for the future development of novel and ultrafast networks of VCSEL-based photonic spiking neurons with brain-inspired connectivity for non-traditional information processing tasks going beyond classical digital systems. 
To further explore the spiking propagation properties between two connected VCSEL-neurons we have investigated the effects of the temporal duration $\left(t_{d}\right)$ of the external perturbations (stimuli) entering T-VCSEL. Figs. 3(e-g) plot measured time series at the outputs of T-VCSEL (top, in blue) and R-VCSEL (bottom, in red) when the length of the incoming perturbation $t_{d}$ is increased from $1.3 \mathrm{~ns}$ to $6.1 \mathrm{~ns}$. For clarity, the traces from T-VCSEL have been shifted upwards by $0.15 \mathrm{mV}$ to plot them together with those from R-VCSEL. The injected signals in each case are showed in the top insets (black) in figs. $3(\mathrm{e}-\mathrm{g})$. The other system parameters are exactly the same as those used in figs. 3(a-d). The top (blue) time traces in figs. 3(eg) show that in all cases the arrival of the external perturbation brings T-VCSEL out of the injection locking range making the device transit into a spiking firing dynamical regime where the number of spikes fired by T-VCSEL grows with increasing $t_{d}$, from 1 (fig. 3(e), $t_{d}=1.3 \mathrm{~ns}$ ) to 4 (fig. 3(f), $t_{d}=4.3 \mathrm{~ns}$ ) and 6 (fig. $\left.3(\mathrm{~g}), \quad t_{d}=6.1 \mathrm{~ns}\right)$ [35]. Thus, simply by controlling experimentally the duration of the encoded perturbations, single or multiple spiking patterns can be generated from the first VCSEL-neuron in the system (T-VCSEL). The bottom plots (in red) in figs. 3(e-g) show in turn the time series measured from the second VCSEL-neuron in the system (R-VCSEL) following the arrival of single and multiple spiking signals from $\mathrm{T}$ VCSEL. All cases depicted in figs. 3(e-g) show that R-VCSEL responds by producing an analogous spike firing pattern to the one arriving from T-VCSEL. As a result, 1, 4 and 6 spikes are respectively obtained at the output of R-VCSEL following the arrival of signals with 1,4 and 6 spikes from T-VCSEL. Moreover, these spikes have similar features, including shape, temporal (sub-ns) duration, intensity and inter-spike intervals. Hence, the arrival of a controlled perturbation encoded in the externally injected signal triggers a transition between injection locking and unlocking states in the interconnected VCSELs and as a result a spike firing pattern is generated and propagated in our proposed system. Additionally, since the two VCSELs used here have similar intrinsic parameters and analogous performance they exhibit similar dynamical responses under the arrival of external stimuli; the slight variations in spike amplitudes observed in fig. 3 are attributed to noise and inherent instabilities in the experimental setup and not to intrinsic response of the system. Fig. 3(e-g) therefore shows the successful communication of both single and multiple spiking information between two interconnected VCSELs. Once again, this is indeed the same behavior as observed in biological neuronal systems but 8 orders of magnitude faster [49-50]. We must note here that the time interval between consecutive events arriving into T-VCSEL will have to be larger than the refractory period [48] of the device for it to be able to yield a desired response. The same applies for the time interval between consecutive spikes arriving from T-VCSEL into RVCSEL. In our case, the spikes generated by T-VCSEL are separated by approx. 1ns which is larger than the expected refractory period [48] and therefore R-VCSEL has time to recover between arriving events yielding a similar spiking pattern as that arriving from T-VCSEL.
It should be pointed out again that there is a time delay between the spiking responses of two VCSEL-neurons in our system. This corresponds to the optical flight time between the two VCSELs in our setup which is of $\sim 62 \mathrm{~ns}$. For simplicity here we have shifted the time series of R-VCSEL in our plots to show all results in a similar temporal scale. Figs. 3(h-i) show the corresponding temporal maps for the results included in Figs. 3(e-g) for T-VCSEL (fig. 3(h)) and R-VCSEL (fig. 3(i)), respectively. Both maps are composed of 9 segments each one of them plotting 20 superimposed time traces for a different value of $t_{d}$ from 0.8 to $6.8 \mathrm{~ns}$ as indicated. Figs. 3(h-i) show that controllable and repeatable spiking patterns with specific number of spikes from 1 to 7 can be obtained from T-VCSEL just by varying the value of $t_{d}$ from 0.8 to $6.8 \mathrm{~ns}$ and are successfully communicated to R-VCSEL.
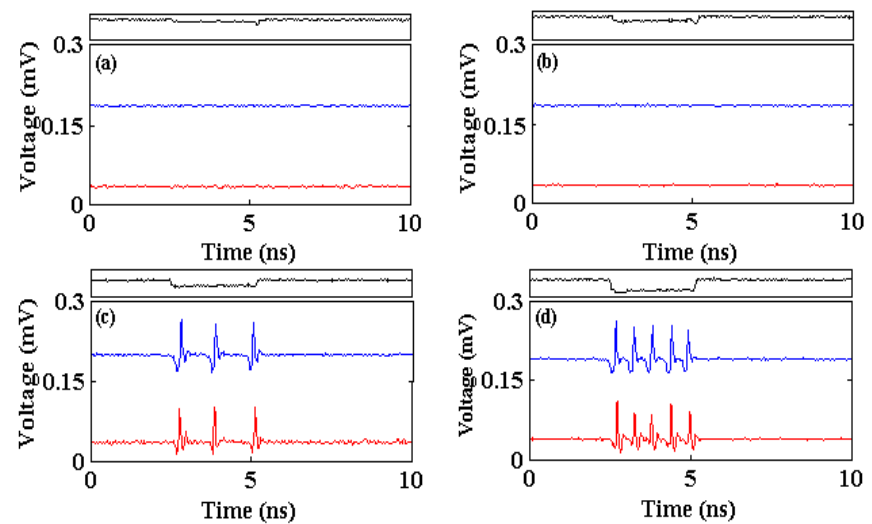

Fig. 4. Time series measured from T-VCSEL (blue) and R-VCSEL (red) for different values of $K_{p}$ : (a) 0.12 , (b) 0.16 , (c) 0.40 and (d) 0.54 . The time duration of the perturbation was set constant at all moments and equal to $t_{d}=4.5 \mathrm{~ns}$. The black insets at the top plot the externally injected optical signal into T-VCSEL

Additionally, we have also investigated the influence of the external perturbations' strength $\left(K_{p}\right)$ on the characteristics of the communication of the spiking information. Fig. 4 plots results for the case where a relative long external perturbation $\left(t_{d}=4.5 \mathrm{~ns}\right)$ arrives into T-VCSEL generating multiple spiking events and which are in turn transmitted to R-VCSEL. For clarity in fig. 4 the measured time series from T-VCSEL (in blue, top) have been shifted upwards by $0.15 \mathrm{mV}$ with respect to those measured for R-VCSEL (in red, bottom). Here, the perturbation's strength $K_{p}$ is increased from 0.12 to 0.54 whilst the perturbation's duration is fixed at $t_{d}=4.5 \mathrm{~ns}$. For the results shown in fig. 4 , the frequency detunings between the external signal and T-VCSEL $(\Delta f)$ and between T-VCSEL and RVCSEL $\left(\Delta f_{T R}\right)$ were set equal to $-3.54 \mathrm{GHz}$ and $-2.84 \mathrm{GHz}$, respectively. Also, the constant injection power level arriving from the external signal into T-VCSEL was equal to $K_{i n j}=68$ $\mu \mathrm{W}$ whilst the constant power level of the signal arriving from T-VCSEL into R-VCSEL was measured equal to $49 \mu \mathrm{W}$. Figs. 4(a) and 4(b) shows that for $K_{p}=0.12$ and 0.16 , the strength of the perturbation is not high enough to yield a spike firing response from T-VCSEL. As a result, T-VCSEL remains in its stable injection locking state and a constant temporal output is obtained at the output of the first VCSEL, as it can be seen from the top diagrams in figs. 4(a) and 4(b). Analogously, since there 
are no spiking events from T-VCSEL entering R-VCSEL, the latter also remains in a stable injection locking state (see bottom diagrams in figs. 4(a) and 4(b)) yielding as well constant output time traces. However, the situation changes when the perturbation's strength is increased. For the case of $K_{p}=0.40$, as seen in the top diagram of fig. 4(c) three spikes are obtained at the output of T-VCSEL given that the perturbation is strong enough to bring the device out of the injection locking state. Now, these spikes fired in T-VCSEL are transmitted along the circuit to R-VCSEL which also reacts by firing three spikes (see bottom diagram in fig. 4(c)). Finally, an analogous response but for a higher number of spikes fired this time is obtained when the perturbation's strength is further grown to higher values. Fig. 4(d) plots the case where $K_{p}$ has been set equal to 0.54 . Here, the strong perturbation yields a five-spikes firing event from T-VCSEL. The latter is successfully transmitted to RVCSEL which also produces a five-spikes firing output. Fig. 4 therefore illustrates first the need for the external perturbation to exceed a critical intensity threshold to generate spike firing events in T-VCSEL and the capability of the generated interconnected VCSEL system to only react and transmit such spiking responses upon the arrival of stimuli of sufficient strength. Additionally, fig. 4 also shows that the number of spikes obtained from T-VCSEL and communicated to RVCSEL can be controllably engineered by varying the value of $K_{p}$ once the exciting threshold is exceeded. This is of importance as it will permit routes to control the spike firing rate in the system not only by acting on the stimulus duration (see fig. 3) but also on the stimulus's strength. Such behavior allowing to compute the information on the arriving stimuli's strength in the generated spike firing rate is also found in biological neurons. Once again, this feature is analogous to that of biological neurons where a clear threshold in stimulus intensity has to be exceed to provoke excitation and firing activity in individual neurons and neuronal circuits. Furthermore, figs. 3 and 4 also reveal that the intensity of the spiking signals obtained from both interconnected VCSELs (Tand R-VCSEL) have a similar level once spiking activity is triggered independently of the strength (providing it exceeds a critical threshold) and temporal duration of the incoming perturbations. This important behavior is also analogous to the all-or-none spike firing response in biological neurons [12].

In this work, we have also investigated numerically the generation and propagation of spiking signals in two interconnected VCSELs. Fig. 5 shows calculated results using the extension of the SFM model described in Section III. Specifically, fig. 5 plots calculated time series from T-VCSEL and R-VCSEL when the former is subject to the arrival of signals with encoded perturbations with different temporal duration ranging from 0.8 ns to $6.8 \mathrm{~ns}$. From these diagrams, it can be seen that single and multiple spikes can be obtained upon the arrival of external perturbations. The higher the perturbation's duration the higher is the number of spikes fired. These diverse spiking dynamics can also be propagated from $\mathrm{T}$ VCSEL to R-VCSEL with the two devices yielding similar spiking responses.

Fig. 5(a) and 5(b) plots respectively calculated time series obtained at the outputs of T-VCSEL (fig. 5(a)) and R-VCSEL (fig. 5(b)). These simulate the response of the devices when an external optical signal with encoded (power drop) perturbations with constant strength ratio of $K_{p}=0.42$ and varying temporal duration $t_{d}$, from 0.8 to $6.8 \mathrm{~ns}$ is injected into T-VCSEL. Fig. 5 shows analogous results to the experimental findings depicted in fig. 3. Here, the arrival of the external perturbations triggers spike firing responses in T-VCSEL as the device goes from being injection locked to unlocked to the external signal.
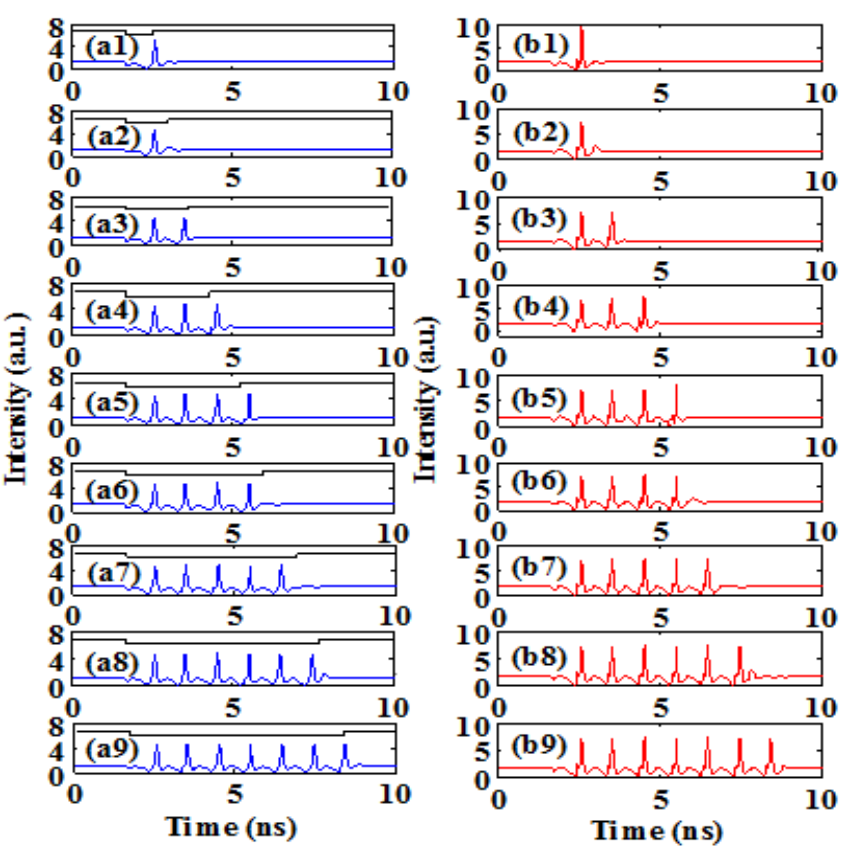

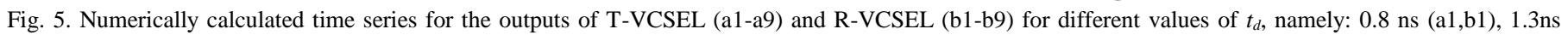

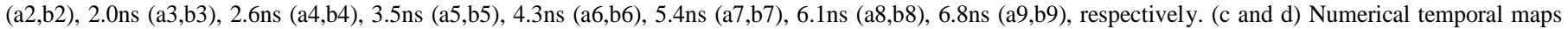
obtained for different values of $t_{d}$, from 0.8 to $6.8 \mathrm{~ns}$ at the outputs of T-VCSEL (c) and R-VCSEL (d). 
Also, as in fig. 3 the number of spikes fired by T-VCSEL increases as the perturbation's duration is grown. Fig. 5(b) plots simulated results for the output of R-VCSEL after the injection of the signals generated by T-VCSEL. Again, as in the experimental findings shown in fig. 3, R-VCSEL responds by reproducing the same spiking pattern arriving from T-VCSEL, firing the same number of spikes with similar intensities, temporal durations and interspike intervals. For simplicity, as in the experimental plots the time series of R-VCSEL have been temporally shifted to plot them in the same scale as those from T-VCSEL. However, it should be noted that the spiking events obtained from R-VCSEL will be obtained after the optical flight time required by T-VCSEL's output to reach R-VCSEL.

Fig. 5(c) and 5(d) show the corresponding calculated temporal maps for the results for T-VCSEL and R-VCSEL respectively. The same colour coding scheme used for the experimental maps of fig. 3 is applied here. The maps merge results calculated from both devices for 9 different values of $t_{d}$ from $0.8 \mathrm{~ns}$ to $6.8 \mathrm{~ns}$ as indicated for the perturbations entering T-VCSEL. For each value of $t_{d}$ the maps plot the response of the system to the arrival of 20 consecutive perturbations. The maps in figs. 5(c) and 5(d) clearly show that the number of spikes obtained from T-VCSEL can be easily controlled by varying the perturbation's duration. Also, the spiking patterns obtained from T-VCSEL are successfully communicated to RVCSEL which reproduces the same spiking patterns. In all cases, the numerical results show that controllable and reproducible spiking signals can be obtained from a first VCSEL-neuron and successfully propagated to a second one, showing indeed an excellent degree of agreement with the experimental findings included in fig. 3. It should be finally noted here that for a better comparison with the experimental findings, only the total output signals from T-VCSEL and RVCSEL are taken into account during the simulation processes, without plotting separately the device's two individual polarizations (parallel and orthogonal). We should mention that the spiking dynamical responses were obtained numerically in the orthogonal polarization output whilst the parallel one remained suppressed. Hence, we should expect the same pattern to occur in the experimental data in future polarization-resolved analysis of the reported spiking regimes.

\section{CONCLUSIONS}

In summary, in this work we demonstrate experimentally and in theory the communication of spiking information between two interconnected 1300nm VCSEL photonic neurons much as biological neurons do in the brain but at sub-nanosecond speeds ( 8 orders of magnitude faster than the millisecond timescales than biological neurons). We show that controllable and reproducible spiking patterns can be obtained in a first VCSELneuron (T-VCSEL) via the external optical injection of signals with added short temporal perturbations. Subsequently, these spiking firing signals from T-VCSEL can be transmitted to a second VCSEL-neuron (R-VCSEL) which responds by reproducing at its output of the same spike firing pattern. Importantly, the characteristics of the two spiking signals obtained from both devices are analogous, showing the same number of spikes fired and interspike time intervals and similar spike intensities. Our results also show that just as biological neurons respond in the brain to incoming stimuli, we can also successfully generate and propagate through the system single (phasic) and multiple (tonic) spiking responses, by simply controlling the temporal duration of the arriving perturbations. Also, a threshold in perturbation's intensity needs to be exceeded to obtain and propagate a spiking response through our photonic neuronal circuit (as it is the case in biological neurons). We have also developed a numerical model which reproduces with high degree of accuracy the experimental findings. Also, very high speed operation (sub-ns speed resolution) and low input power requirements (only a few tens of $\mu$ Watts) were needed in our experiments. These results added to the use of inexpensive commercially available VCSELs operating at the telecom wavelength of $1300 \mathrm{~nm}$, hence totally compatible with optical networking technologies, pave the way towards the realization of interconnected ultrafast networks of photonic spiking neurons with neuro-inspired connectivity. These offer exciting prospects for future advanced neuromorphic photonic modules for use in non-traditional computing paradigms beyond present digital systems.

\section{REFERENCES}

[1] A. S. Cassidy, J. Georgiou, and A. G. Andreou, "Design of silicon brains in the nano-CMOS era: spiking neurons, learning synapses and neural architecture optimization," Neural Networks, vol. 45, pp. 4-26, Sep. 2013.

[2] X. Y. Wu, V. Saxena, K. H. Zhu, and S. Balagopal, "A CMOS spiking neuron for brain-inspireed neural networks neural networks with resistive synapses and in-situ learning," IEEE Trans. on Circuits and Syst. II: Express Briefs, vol. 62, no. 11, pp. 1088-1092, Nov. 2015.

[3] N. Imam, F. Akopyan, J. Arthur, P. Merolla, R. Manohar, and D. S. Modha, "A digital neurosynaptic core using event-driven QDI circuits," IEEE $18^{\text {th }}$ International Symposium on Asynchronous Circuits and Systems, pp. 2532, 7-9 May 2012.

[4] G. Indiveri, B. Linares-Barranco, T. J. Hamilton, André van Schaik, R. Etienne-Cummings, T. Delbruck et al., "Neuromorphic silicon neuron circuits," Frontiers Neurosci., vol. 5, no. 5, Art. ID. 73, May, 2011.

[5] R. J. Vogelstein, U. Mallik, J. T. Vogelstein, and G. Cauwenberghs, "Dynamically reconfigurable silicon array of spiking neurons with conductance-based synapses," IEEE Trans. Neural Netw., vol. 18, no. 1, pp. 253-265, Jan. 2007.

[6] G. Indiveri, E. Chicca, and R. Douglas, "A VLSI array of low-power spiking neurons and bistable synapses with spike-timing dependent plasticity," IEEE Trans. Neural Netw., vol. 17, no. 1, pp. 211-221, Jan. 2006.

[7] B. V. Benjamin, P. Gao, E. McQuinn, S. Choudhary, A. R. Chandrasekaran, Jean-Marie Bussat, et al., "Neurogrid: a mixed-analog-digital multichip system for large-scale neural simulations," Proceedings of the IEEE, vol. 102, no. 5, pp. 699-716, May, 2014.

[8] J. Hsu, "IBM's new brain," IEEE spectrum, vol. 51, no. 10, pp. 17-19, Oct. 2014.

[9] M. M. Khan, D. R. Lester, L. A. Plana, A. Rast, X. Jin, E. Painkras et al., "SpiNNaker: mapping neural networks onto a massively-parallel chip multiprocessor," 2008 IEEE International Joint Conference on Neural Networks (IEEE World Congress on Computational Intelligence), pp. 2849-2856, Jun. 2008.

[10] B. J. Shastri, A. N. Tait, M. A. Nahmias, and P. R. Prucnal, "Photonic spike processing: ultrafast laser neurons and an integrated photonic network," IEEE Photon. Soc. Newslett., vol. 28, no. 3, pp. 4-11, Jun. 2014.

[11] M. P. Fok, Y. Tian, D. Rosenbluth, and P. R. Prucnal, "Asynchronous spiking photonic neuron for lightwave neuromorphic signal processing," Opt. Lett., vol. 37, no. 16, pp. 3309-3311, Aug. 2012.

[12] P. R. Prucnal, B. J. Shastri, T. F. Lima, M. A. Nahmias, and A. N. Tait, 
"Recent progress in semiconductor excitable lasers for photonic spike processing," Advances in Optics and Photon., vol. 8, no. 2, pp. 228-299, Jun. 2016.

[13] W. Coomans, L. Gelens, S. Beri, J. Danckaert, and G. Van der Sande, "Solitary and coupled semiconductor ring lasers as optical spiking neurons," Phys. Rev. E, vol. 84, no. 3, Art. ID. 036209, Sep. 2011.

[14] K. Kravtsov, M. P. Fok, D. Rosenbluth, and P. R. Prucnal, "Ultrafast alloptical implementation of a leaky integrate-and-fire neuron," Opt. Express, vol. 19, no. 3, pp. 2133-2147, Jan. 2011.

[15] B. J. Shastri, M. A. Nahmias, A. N. Tait, A. W. Rodriguez, B. Wu and P. R. Prucnal, "Spike processing with a graphene excitable laser," Sci. Rep., vol. 6, Art. ID. 19126, Jan. 2016.

[16] L. A. Coldren, S. W. Corzine, and M. L. Mashanovitch, Diode Lasers and Photonic Integrated Circuits (John Wiley \& Sons, 2012).

[17] B. Kelleher, C. Bonatto, G. Huyet, and S. P. Hegarty, "Excitability in optically injected semiconductor lasers: contrasting quantum-well- and quantum-dot-based devices," Phys. Rev. E, vol. 83, no. 2, Art. ID. 026207, Feb. 2011.

[18] H. J. Wünsche, O. Brox, M. Radziunas, and F. Henneberger, "Excitability of a semiconductor laser by a two-mode homoclinic bifurcation," Phys. Rev. Lett., vol. 88, no. 2, Art. ID. 023901, Jan. 2002.

[19] S. Wieczorek, B. Krauskopf, and D. Lenstra, "Multipulse excitability in a semiconductor laser with optical injection," Phys. Rev. Lett., vol. 88, no. 6, Art. ID. 063901, Feb. 2002.

[20] I. Aldaya, C. Gosset, C. Wang, G. Campuzano, F. Grillot, and G. Castañon, "Periodic and aperiodic pulse generation using optically injected DFB lasers," Electron. Lett., vol. 51, no. 3, pp. 280-282, Feb. 2015.

[21] A. Aragoneses, S. Perrone, T. Sorrentino, M. C. Torrent, and C. Masoller, "Unveiling the complex organization of recurrent patterns in spiking dynamical systems," Sci. Rep., vol. 4, Art. ID. 04696, Apr. 2014.

[22] S. Barbay, R. Kuszelewicz, and A. M. Yacomotti, "Excitability in a semiconductor laser with saturable absorber," Opt. Lett., vol. 36, no. 23, pp. 4476-4478, Dec. 2011.

[23] A. N. Tait, M. A. Nahmias, B. J. Shastri, and P. R. Prucnal, "Broadcast and weight: an integrated network for scalable photonic spike processing," IEEE J. Lightwave Technol., vol. 32, no, 21, pp. 4029-4041, Nov. 2014.

[24] C. Mesaritakis, A. Kapsalis, A. Bogris, and D. Syvridis, "Artificial neuron based on integrated semiconductor quantum dot mode-locked lasers," Sci. Rep., vol. 6, Art. ID. 39317, Dec. 2016.

[25] A. M. Yacomotti and G. B. Mindlin, "Coupled optical excitable cells," Phys. Rev. E, vol, 66, no. 3, Art. ID. 036227, Sep. 2002.

[26] B. Kelleher, C. Bonatto, P. Skoda, S. P. Hegarty, and G. Huyet, "Excitation regeneration in delay-coupled oscillators," Phys. Rev. E, vol. 81, no. 3, Art. ID. 036204, Mar. 2010.

[27] T. V. Vaerenbergh, K. Alexander, J. Dambre and P. Bienstman, "Excitation transfer between optically injected microdisk lasers," Opt. Express, vol. 21, no. 23, pp. 28922-28932, Nov. 2013.

[28] T. V. Vaerenbergh, M. Fiers, P. Mechet, T. Spuesens, R. Kumar, G. Morthier et al., "Cascadable excitability in microrings," Opt. Express, vol. 20, no. 18, pp. 20292-20308, Aug. 2012.

[29] K. Iga, "Surface-emitting laser-its birth and generation of new optoelectronics field," IEEE J. Sel. Top. Quantum Electron., vol. 6, no. 6, pp. 1201-1215, Nov./Dec. 2000.

[30] F. Koyama, "Recent advances of VCSEL photonics," IEEE J. Lightwave Technol., vol. 24, no. 12, pp. 4502-4513, Dec. 2006.

[31] M. J. Adams, A. Hurtado, D. Labukhin, and I. D. Henning, "Nonlinear semiconductor lasers and amplifiers for all-optical information processing," Chaos, vol. 20, no. 3, Art. ID. 037102, Sep. 2010.

[32] K. Schires, A. Hurtado, I. D. Henning and M. J. Adams, "Rare disruptive events in polarization-resolved dynamics of optically injected $1550 \mathrm{~nm}$ VCSELs," Electron. Lett., vol. 48, no. 14, pp. 872-873, Jul. 2012.

[33] S. Y. Xiang, A. J. Wen and W. Pan, "Emulation of spiking response and spiking frequency property in VCSEL-based photonic neuron," IEEE Photon. J., vol. 8, no. 5, Art. ID. 1504109, Oct. 2016.

[34] M. Turconi, B. Garbin, M. Feyereisen, M. Giudici, and S. Barland, "Control of excitable pulses in an injection-locked semiconductor laser," Phys. Rev. E., vol. 88, no. 2, Art. ID. 022923, Aug. 2013.

[35] A. Hurtado, and J. Javaloyes, "Controllable spiking pattern in longwavelength vertical surface emitting lasers for neuromorphic photonics systems," Appl. Phys. Lett., vol. 107, no. 24, Art. ID. 241103, Dec. 2015.

[36] Y. Onishi, N. Nishiyama, C. Caneau, F. Koyama, and C. E. Zah, "Alloptical inverter based on long-wavelength vertical-cavity surfaceemitting laser," IEEE J. Sel. Top. Quantum Electron., vol. 11, no. 5, pp. 999-1005, Sep./Oct. 2005.
[37] J. Sakaguchi, T. Katayama, and H. Kawaguchi, "All-optical memory operation of 980-nm polarization bistable VCSEL for $20-\mathrm{Gb} / \mathrm{s}$ PRBS RZ and 40-Gb/s NRZ data signals," Opt. Express, vol. 18, no. 12, pp. $12362-$ 12370, Jun. 2010.

[38] S.-H. Lee, H.-W. Jung, K.-H. Kim, and M.-H. Lee, "All-optical flip-flop operation based on polarization bistability of conventional-type $1.55-\mu \mathrm{m}$ wavelength single-mode VCSELs," J. Opt. Soc. Korea, vol. 14, no. 2, pp. 137-141, Jun. 2010.

[39] B. Garbin, J. Javaloyes, G. Tissoni, and S. Barland, "Topological solitons as addressable phase bits in a driven laser," Nat. Commun., vol. 6, Art. ID. 5915, Jan. 2015

[40] B. Jiang, Z. M. Wu, T. Deng, J. J. Chen, F. Yang, J. Chen et al., "Polarization switching characteristics of 1550-nm vertical-cavity surface-emitting lasers subject to double polarization pulsed injection," IEEE J. Quantum Electron., vol. 52, no. 11, Art. ID. 2400707, Nov. 2016.

[41] P. Pérez, A. Valle, L. Pesquera, and A. Quirce, "All-Optical inverter based on polarization switching in VCSELs subject to single and dual optical injection," IEEE J. Sel. Top. Quantum Electron., vol. 19, no. 4, Art. ID. 1700408, Jul./Aug. 2013.

[42] A. Hurtado, I. D. Henning, and M. J. Adams, "Optical neuron using polarization switching in a $1550 \mathrm{~nm}$-VCSEL," Opt. Express, vol. 18, no. 24, pp. 25170-25176, Nov. 2010.

[43] A. Hurtado, K. Schires, I. D. Henning and M. J. Adams, "Investigation of vertical cavity surface emitting laser dynamics for neuromorphic photonic systems," Appl. Phys. Lett., vol. 100, no. 10, Art. ID. 103703, Mar. 2012.

[44] R. Al-Seyab, K. Schires, N. A. Khan, A. Hurtado, I. D. Henning, and M. J. Adams, "Dynamics of polarized optical injection in 1550-nm VCSELs: theory and experiments," IEEE J. Sel. Top. in Quantum Electron., vol. 17, no. 5, pp. 1242-1249, Sep./Oct. 2011.

[45] P. Pérez, A. Valle, I. Noriega, and L. Pesquera, "Measurement of the intrinsic parameters of single-mode VCSELs," IEEE J. Lightwave Technol., vol. 32, no. 8, pp. 1601-1607, Apr. 2014.

[46] A. Hurtado, D. Labukhin, I. D. Henning, and M. J. Adams, "Injection locking bandwidth in 1550-nm VCSELs subject to parallel and orthogonal optical injection," IEEE J. Sel. Top. in Quantum Electron., vol. 15, no. 3, pp. 585-593, May/Jun. 2009.

[47] P. Pérez, A. Quirce, L. Pesquera, and A. Valle, "Polarization-resolved nonlinear dynamics induced by orthogonal optical injection in longwavelength VCSELs," IEEE J. Sel. Top. in Quantum Electron., vol. 17, no. 5, pp. 1228-1235, Sep./Oct. 2011.

[48] B. Garbin, A. Dolcemascolo, F. Prati, J. Javaloyes, G. Tissoni, and S. Barland, "Refractory period of an excitable semiconductor laser with optical injection," Phys. Rev. E, vol. 95, no. 1, Art. ID. 012214, Jan. 2017.

[49] M. Diesmann, Marc-Oliver Fewaltig, and A. Aertsen, "Stable propagation of synchronous spiking in cortical neural networks," Nature, vol. 402, pp. 529-533, Dec. 1999.

[50] G. Schneider, D. Nikolié, "Detection and assessment of near-zero delays in neuronal spiking activity," J. Neurosci. Methods, vol. 152, no. 1-2, pp. 97-106, Apr. 2006.

Tao Deng was born in Sichuan, China, in 1982. He received the B.Sc. degree in electronic information engineering and the M.Sc. degrees in optics from Southwest University, Chongqing, China, in 2002, 2005, and the Ph. D. degree in optics from Sichuan University, Chengdu, China, in 2012. He is currently an associate Professor with the School of Physical Science and Technology, Southwest University, Chongqing. He has authored or co-authored over 30 publications. His current research mainly focuses on the nonlinear dynamics of semiconductor lasers and their applications in chaos secret communication, microwave photonics, photonic neuron etc.

Joshua Robertson was born in Glasgow (UK) in 1994. He is working towards the M.Sc. degree in Physics from the University of Strathclyde (Glasgow).

Antonio Hurtado received the $\mathrm{PhD}$ degree from the Universidad Politécnica de Madrid (UPM), Spain in 2006. He has over 10 years' international research experience in photonics in the UK (Universities of Essex and Strathclyde), USA (University of New Mexico) and Spain (UPM). He has been awarded two prestigious and highly competitive Marie Curie Fellowships by the European Commission: Projects ISLAS (2009-2011) \& NINFA (2011-2014). In 2014 he was awarded a Strathclyde Chancellor's Fellowship following which he was appointed as Lecturer at the Strathclyde's Institute of Photonics. 